\title{
UNIQUENESS THEOREMS FOR SOME FOURTH-ORDER ELLIPTIC EQUATIONS
}

\author{
ROBERT DALMASSO \\ (Communicated by Barbara Lee Keyfitz)
}

\begin{abstract}
The purpose of this paper is to prove the uniqueness of positive solutions of some particular biharmonic boundary value problems. We also give some existence results in the sublinear case.
\end{abstract}

\section{INTRODUCTION}

In this paper we consider the Dirichlet problem

$$
\begin{cases}\Delta^{2} u=|u|^{p} & \text { in } B_{R} \\ u=\frac{\partial u}{\partial \nu}=0 & \text { on } \partial B_{R}\end{cases}
$$

and the Navier problem

$$
\begin{cases}\Delta^{2} u=|u|^{p} & \text { in } B_{R}, \\ u=\Delta u=0 & \text { on } \partial B_{R},\end{cases}
$$

where $B_{R}$ denotes the ball of radius $R$ centered at the origin in $\mathbb{R}^{n}(n \geq 1)$, $\partial B_{R}$ is the boundary of $B_{R}, \frac{\partial}{\partial \nu}$ is the outward normal derivative, and $p \in$ $(0,1) \cup(1,+\infty)$.

We are interested in the uniqueness question for problems (1.1) and (1.2). Our main results are the following two theorems.

Theorem 1.1. (i) Suppose $n \geq 2$. Let $u \in C^{4}\left(\bar{B}_{R}\right)$ be a nontrivial radial solution of (1.1). Then $u>0$ in $B_{R}$ and $u$ is the unique nontrivial radial solution of (1.1) in $C^{4}\left(\bar{B}_{R}\right)$.

(ii) Suppose $n=1$. Let $u \in C^{4}([-R, R])$ be a nontrivial solution of $(1.1)$. Then $u>0$ in $(-R, R)$ and $u$ is the unique nontrivial solution of (1.1) in $C^{4}([-R, R])$.

Theorem 1.2. Let $u \in C^{4}\left(\bar{B}_{R}\right)$ be a nontrivial solution of (1.2). Then $u>0$ in $B_{R}$ and $u$ is the unique nontrivial solution of $(1.2)$ in $C^{4}\left(\bar{B}_{R}\right)$.

When $1 \leq n \leq 3$ and $p>1$ the uniqueness of a positive radial solution of problem (1.1) was proved by Dunninger and Miklavcic [3]. When $n=1$

Received by the editors July 14, 1993.

1991 Mathematics Subject Classification. Primary 35J65.

Key words and phrases. Semilinear biharmonic B.V.P., maximum principle. 
and $p>1$, the uniqueness of a positive solution of problem (1.1) was established in [2] where we first showed that positive solutions of problem (1.1) were symmetric about the origin. As mentioned in that paper the same proof gives the uniqueness of a positive radial solution for $n \geq 2$ and $p>1$. In the present paper we give a simpler proof. When $0<p<1$ we do not know any uniqueness results and the proof given in [2] for $p>1$ does not hold. As for problem (1.2) a uniqueness result for positive radial solutions was obtained by Peletier and van der Vorst [4] when $p>1$. Our proof below is simpler. When $p>1$ the existence of a positive solution $u \in C^{4}\left(\bar{B}_{R}\right)$ of (1.1) was established in [1] where more general nonlinearities are studied (actually, only the case $n \geq 5$ was considered in [1]). Moreover, we proved in [1] that, when $n \geq 5$ and $p \geq(n+4) /(n-4),(1.1)$ has no positive solution whether radial or not. For problem (1.2) the existence of a positive solution was established in [4] when $p>1$. The existence of a positive solution of (1.1) (resp. (1.2)) in the sublinear case $0<p<1$ is easily obtained. Since we have not been able to find a proof in the literature, we will discuss it briefly in the last section.

In the sequel $\Delta$ denotes equally the cartesian form and the polar form of the Laplacian.

In $\S 2$ we give some lemmas which are needed in the proof of the theorems, in particular, to establish the existence results. Theorems 1.1 and 1.2 are proved in $\S 3$. Finally in $\S 4$ we give some existence results in the sublinear case.

\section{PRELIMINARIES}

The Green's function of the operator $\Delta^{2}$ for the Dirichlet problem in $[0, R)$ can be explicitly determined.

Lemma 2.1. The Green's function of the operator $\Delta^{2}$ for the Dirichlet problem in $[0, R)$ is given by $K_{n}(t, s)=R^{3} L_{n}\left(\frac{t}{R}, \frac{s}{R}\right)$, where

$$
L_{n}(t, s)= \begin{cases}X_{n}(s)+t^{2} Y_{n}(s), & 0 \leq t \leq s \leq 1, \\ \left(\frac{s}{t}\right)^{n-1}\left(X_{n}(t)+s^{2} Y_{n}(t)\right), & 0 \leq s \leq t \leq 1,\end{cases}
$$

with

$$
X_{n}(t)= \begin{cases}\frac{t-t^{3}(1-2 \ln t)}{8} & \text { if } n=2, \\ \frac{t^{5}-2 t^{3} \ln t-t^{3}}{8} & \text { if } n=4, \\ \frac{t^{3}}{4(n-2)(n-4)}\left((n-4) t^{n-2}-(n-2) t^{n-4}+2\right) & \text { if } n \neq 2,4\end{cases}
$$

and

$$
Y_{n}(t)= \begin{cases}\frac{t(1+2 \ln t)-t^{3}}{8} & \text { if } n=2, \\ \frac{-t^{5}+2 t^{3}-t}{16} & \text { if } n=4, \\ \frac{t}{4 n(n-2)}\left(n t^{n-2}-(n-2) t^{n}-2\right) & \text { if } n \neq 2,4 .\end{cases}
$$

Moreover, $K_{n}$ has the following properties:

$$
\begin{gathered}
\frac{\partial}{\partial t} K_{n}(t, s) \leq 0 \text { for } t, s \in[0, R], \\
\frac{\partial^{2}}{\partial t^{2}} K_{n}(R, s)=\frac{R}{2}\left(\frac{s}{R}\right)^{n-1}\left(1-\left(\frac{s}{R}\right)^{2}\right) \quad \text { for } s \in[0, R] .
\end{gathered}
$$

The detailed verification of this lemma is left to the reader. 
Lemma 2.2. Let $u \in C^{4}([0, R])$ be such that $\Delta^{2} u>0$ on $[0, R), u(R)=$ $u^{\prime}(R)=0$. Then $u^{\prime}<0$ on $(0, R)$ and $(\Delta u)^{\prime}>0$ on $(0, R]$. Furthermore, $(\Delta u)(0)<0$ and $(\Delta u)(R)=u^{\prime \prime}(R)>0$.

Proof. Using Lemma 2.1 we deduce that $u>0$ on $[0, R)$. If $\Delta u \geq 0$ in $[0, R]$, since $u(R)=0$, the maximum principle gives a contradiction. Now, if $\Delta u \leq 0$ in $[0, R]$, since $u(R)=u^{\prime}(R)=0$, the Hopf boundary lemma implies that $u=0$ in $[0, R]$ and we again reach a contradiction. Since

$$
t^{n-1}(\Delta u)^{\prime}(t)=\int_{0}^{t} s^{n-1}\left(\Delta^{2} u\right)(s) d s>0 \text { for } t \in(0, R],
$$

we conclude that $(\Delta u)(0)<0$ and $(\Delta u)(R)=u^{\prime \prime}(R)>0$. Then, the fact that $u^{\prime}<0$ on $(0, R)$ follows easily from the maximum principle.

The next lemma is an immediate consequence of Lemma 2.1.

Lemma 2.3. Let $g \in C([0, R])$ be such that $g \geq 0$ and $g \not \equiv 0$. Then the function

$$
h(t)=\int_{0}^{R} K_{n}(t, s) g(s) d s, \quad 0 \leq t \leq R,
$$

is such that $h(t) \geq C(R-t)^{2}$ for $0 \leq t \leq R$, where $C>0$ is a constant.

The Green's function of the operator $-\Delta$ for the Dirichlet problem in $[0, R)$ is given by $G_{n}(t, s)=R H_{n}\left(\frac{t}{R}, \frac{s}{R}\right)$, where

$$
\begin{aligned}
& H_{1}(t, s)= \begin{cases}1-s, & 0 \leq t \leq s \leq 1, \\
1-t, & 0 \leq s \leq t \leq 1,\end{cases} \\
& H_{2}(t, s)= \begin{cases}-s \ln s, & 0 \leq t \leq s \leq 1, \\
-s \ln t, & 0 \leq s \leq t \leq 1,\end{cases}
\end{aligned}
$$

and

$$
H_{n}(t, s)= \begin{cases}\frac{1}{n-2} s\left(1-s^{n-2}\right), & 0 \leq t \leq s \leq 1, \\ \frac{1}{n-2} s^{n-1}\left(t^{2-n}-1\right), & 0 \leq s \leq t \leq 1,\end{cases}
$$

if $n \geq 3$.

We easily prove the following lemma.

Lemma 2.4. Let $g \in C([0, R])$ be such that $g \geq 0$ and $g \not \equiv 0$. Then the function

$$
h(t)=\int_{0}^{R} G_{n}(t, s) g(s) d s, \quad 0 \leq t \leq R,
$$

is such that $h(t) \geq C(R-t)$ for $0 \leq t \leq R$, where $C>0$ is a constant.

\section{PROOF OF THE THEOREMS}

Proof of Theorem 1.1. (i) Let $u \in C^{4}\left(\bar{B}_{R}\right)$ be a nontrivial radial solution of (1.1). Define $y(|x|)=u(x)$ for $x \in \bar{B}_{R}$. Using Lemma 2.1 we deduce that $y>0$ on $[0, R)$. By Lemma 2.2 we have $y^{\prime}<0$ on $(0, R)$ and $(\Delta y)^{\prime}>0$ on $(0, R]$. Let $v \in C^{4}\left(\bar{B}_{R}\right)$ be another nontrivial radial solution of (1.1). Define $z(|x|)=v(x)$ for $x \in \bar{B}_{R}$. Then $z>0$ on $[0, R), z^{\prime}<0$ on $(0, R)$, and $(\Delta z)^{\prime}>0$ on $(0, R]$. 
Let $\lambda^{4 /(p-1)}=y(0) / z(0)$, and define the function

$$
w(t)=\lambda^{4 /(p-1)} z(\lambda t) \quad \text { for } t \in[0, R / \lambda] .
$$

Clearly we have

$$
\left\{\begin{array}{l}
\Delta^{2} w=w^{p} \quad \text { in }[0, R / \lambda) \\
w(R / \lambda)=w^{\prime}(R / \lambda)=0
\end{array}\right.
$$

and

$$
y(0)=w(0)
$$

We first prove that

$$
\Delta y(0)=\Delta w(0)
$$

Suppose that $\Delta y(0)<\Delta w(0)$. Define $R(\lambda)=\min (R, R / \lambda)$. If there exists $a \in(0, R(\lambda)]$ such that $\Delta(y-w)<0$ on $[0, a)$ and $\Delta(y-w)(a)=0$, then the maximum principle and (3.1) imply that $(y-w)(t)<0$ for $t \in(0, a$ ] Therefore, $\Delta^{2}(y-w)=y^{p}-w^{p}<0$ on $(0, a]$ and the maximum principle implies that $\Delta(y-w)>\Delta(y-w)(a)=0$ on $[0, a)$, a contradiction. Thus $\Delta(y-w)<0$ on $[0, R(\lambda)]$. Then, as before, we show that $(y-w)(t)<0$ for $t \in(0, R(\lambda)]$. Since

$$
(y-w)(R(\lambda))= \begin{cases}y(R / \lambda) & \text { if } \lambda>1, \\ 0 & \text { if } \lambda=1, \\ -w(R) & \text { if } \lambda<1,\end{cases}
$$

we deduce that necessarily $\lambda<1$. Now the Hopf boundary lemma implies that $(y-w)^{\prime}(R)<0$. Since $(y-w)^{\prime}(R)=-w^{\prime}(R)=-\lambda^{(p+3) /(p-1)} z^{\prime}(\lambda R)$, we again obtain a contradiction. The case $\Delta y(0)>\Delta w(0)$ can be handled in the same way. Thus (3.2) is proved.

Now we define the functions $Y, W$, and $F$ by

$$
\begin{array}{cl}
Y(t)=(y(t), \Delta y(t)) & \text { for } t \in[0, R], \\
W(t)=(w(t), \Delta w(t)) & \text { for } t \in[0, R / \lambda],
\end{array}
$$

and

$$
F(s, t)=\left(t, s^{p}\right) \text { for } s \geq 0, t \in \mathbb{R} .
$$

Using (3.1), (3.2), and the fact that $y^{\prime}(0)=w^{\prime}(0)=y^{\prime \prime \prime}(0)=w^{\prime \prime \prime}(0)=0$ we easily obtain

$$
\begin{aligned}
& Y(t)-W(t)=\frac{1}{n-2} \int_{0}^{t}\left(1-\left(\frac{s}{t}\right)^{n-2}\right) s(F(Y(s))-F(W(s))) d s \quad \text { when } n \geq 3, \\
& Y(t)-W(t)=\int_{0}^{t} s \ln \left(\frac{t}{s}\right)(F(Y(s))-F(W(s))) d s \quad \text { when } n=2, \\
& Y(t)-W(t)=\int_{0}^{t}(t-s)(F(Y(s))-F(W(s))) d s \quad \text { when } n=1
\end{aligned}
$$

for $t \in[0, R(\lambda)]$. When $p>1, F$ is locally Lipschitz continuous, and using Gronwall's lemma we obtain $Y=W$ on $[0, R(\lambda)]$. When $0<p<1$ let $a \in(0, R(\lambda))$ be fixed. Then $y(0) \geq y(s) \geq y(a)>0$ and $w(0)=y(0) \geq$ $w(s) \geq w(a)>0$ for $s \in[0, a]$. Since $F$ is locally Lipschitz continuous on $(0,+\infty) \times \mathbb{R}$, as before we obtain $Y=W$ on $[0, a]$. By continuity we get 
$Y=W$ on $[0, R(\lambda)]$. Now we deduce that $\lambda=1$ and thus $y=z$ on $[0, R]$. The proof of (i) is complete.

(ii) Let $u \in C^{4}([-R, R])$ be a nontrivial solution of (1.1). By Theorem 1.1 in [2] we know that $u$ is symmetric about the origin, $u^{\prime}<0$ on $(0, R)$, and $u^{\prime \prime \prime}>0$ on $(0, R]$. Then the proof is the same as in (i).

Proof of Theorem 1.2. Let $u \in C^{4}\left(\bar{B}_{R}\right)$ be a nontrivial solution of (1.2). Using the maximum principle we easily get $u>0$ on $B_{R}$ and $\Delta u<0$ on $B_{R}$. Then it is well known (see Troy [5]) that $u$ is radially symmetric and that if $y(|x|)=u(x)$, we have $y^{\prime}<0$ on $(0, R)$ and $(\Delta y)^{\prime}>0$ on $(0, R)$. Let $v \in C^{4}\left(\bar{B}_{R}\right)$ be another nontrivial solution of (1.2). Then $v>0$ on $B_{R}$, $\Delta v<0$ on $B_{R}, v$ is radially symmetric, and if $z(|x|)=v(x)$, we have $z^{\prime}<0$ on $(0, R)$ and $(\Delta z)^{\prime}>0$ on $(0, R)$.

Let $\lambda^{4 /(p-1)}=y(0) / z(0)$, and define the function

$$
w(t)=\lambda^{4 /(p-1)} z(\lambda t) \text { for } t \in[0, R / \lambda] .
$$

Clearly we have

$$
\left\{\begin{array}{l}
\Delta^{2} w=w^{p} \quad \text { in }[0, R / \lambda) \\
w(R / \lambda)=w^{\prime \prime}(R / \lambda)=0
\end{array}\right.
$$

and

$$
y(0)=w(0)
$$

We first prove that

$$
\Delta y(0)=\Delta w(0)
$$

Suppose that $\Delta y(0)<\Delta w(0)$. If there exists $a \in(0, R(\lambda)]$ such that $\Delta(y-w)<0$ on $[0, a)$ and $\Delta(y-w)(a)=0$, then the maximum principle and (3.3) imply that $(y-w)(t)<0$ for $t \in(0, a]$. Therefore, $\Delta^{2}(y-w)=$ $y^{p}-w^{p}<0$ on $(0, a]$ and the maximum principle implies that $\Delta(y-w)>$ $\Delta(y-w)(a)=0$ on $[0, a)$, a contradiction. Thus $\Delta(y-w)<0$ on $[0, R(\lambda)]$. Since

$$
\Delta(y-w)(R(\lambda))= \begin{cases}\Delta y(R / \lambda) & \text { if } \lambda>1, \\ 0 & \text { if } \lambda=1, \\ -\Delta w(R) & \text { if } \lambda<1,\end{cases}
$$

we deduce that necessarily $\lambda>1$. Now as before, we show that $(y-w)(t)<0$ for $t \in(0, R(\lambda)]$. Since

$$
(y-w)(R(\lambda))= \begin{cases}y(R / \lambda) & \text { if } \lambda>1, \\ 0 & \text { if } \lambda=1, \\ -w(R) & \text { if } \lambda<1,\end{cases}
$$

we deduce that necessarily $\lambda<1$ and obtain a contradiction. The case $\Delta y(0)>$ $\Delta w(0)$ can be handled in the same way. Thus (3.4) is proved. Then we conclude as in the proof of Theorem 1.1.

\section{EXISTENCE RESUltS When $0<p<1$}

We consider the more general problems

$$
\begin{cases}\Delta^{2} u=f(u) & \text { in } B_{R} \\ u=\frac{\partial u}{\partial \nu}=0 & \text { on } \partial B_{R}\end{cases}
$$


and

$$
\begin{cases}\Delta^{2} u=f(u) & \text { in } B_{R} \\ u=\Delta u=0 & \text { on } \partial B_{R}\end{cases}
$$

where $f: \mathbb{R} \rightarrow \mathbb{R}$ is a continuous function satisfying the following hypotheses:

$\left(\mathrm{H}_{1}\right) \quad f(0)=0, f(u)>0$ for $u>0$ and $f$ is nondecreasing on $[0,+\infty)$.

$\left(\mathbf{H}_{2}\right) \lim _{u \rightarrow 0^{+}} f(u) / u=+\infty$ and $\lim _{u \rightarrow+\infty} f(u) / u=0$.

Theorem 4.1. Problem (4.1) (resp. (4.2)) has at least one positive radial solution $u \in C^{4}\left(\bar{B}_{R}\right)$.

Proof. Since we are interested in positive radial solutions, the problems under consideration reduce to the one-dimensional boundary value problems

$$
\left\{\begin{array}{l}
\Delta^{2} u=f(u) \quad \text { in }[0, R) \\
u(R)=u^{\prime}(R)=0
\end{array}\right.
$$

and

$$
\left\{\begin{array}{l}
\Delta^{2} u=f(u) \quad \text { in }[0, R) \\
u(R)=u^{\prime \prime}(R)=0
\end{array}\right.
$$

We first consider problem $\left(4.1^{\prime}\right)$. By Lemma 2.3 there exists $C>0$ such that

$$
\int_{0}^{R} K_{n}(t, s)(R-s)^{2} d s \geq C(R-t)^{2} \quad \text { for } 0 \leq t \leq R .
$$

Let $M>0$ be such that $M C \geq 1$. By $\left(\mathrm{H}_{2}\right)$ there exist $\rho>\eta>0$ such that $f(u) \geq M u$ for $0 \leq u \leq \eta$ and $f(u) \int_{0}^{R} K_{n}(0, s) d s \leq u$ for $u \geq \rho$. Choose $a, b>0$ in such a way that $a R^{2} \leq \eta<\rho \leq b$. Now let

$$
Z=\left\{u \in C([0, R]) ; a(R-t)^{2} \leq u(t) \leq b \text { for } 0 \leq t \leq R\right\} .
$$

Clearly, $Z$ is a bounded closed convex subset of the Banach space $C([0, R])$ endowed with the sup norm. Define

$$
\dot{T} u(t)=\int_{0}^{R} K_{n}(t, s) f(u(s)) d s
$$

for $t \in[0, R]$ and $u \in Z$. Using $\left(\mathrm{H}_{1}\right)$ and Lemma 2.1 it is easily verified that $T$ is a compact operator mapping $Z$ into itself, and so there exists $u \in Z$ such that $u=T u$ by the Schauder fixed-point theorem. Clearly $u \in C^{4}([0, R])$ and $u$ is a solution of $\left(4.1^{\prime}\right)$.

Now we consider problem $\left(4.2^{\prime}\right)$. By Lemma 2.4 there exists $C>0$ such that

$$
\int_{0}^{R} G_{n}(t, s)(R-s) d s \geq C(R-t) \quad \text { for } 0 \leq t \leq R .
$$

Let $M>0$ be such that $M C^{2} \geq 1$. By $\left(\mathrm{H}_{2}\right)$ there exist $\rho>\eta>0$ such that $f(u) \geq M u$ for $0 \leq u \leq \eta$ and $f(u)\left(\int_{0}^{R} G_{n}(0, s) d s\right)^{2} \leq u$ for $u \geq \rho$. Choose $a, b>0$ in such a way that $a R \leq \eta<\rho \leq b$. Now let

$$
W=\{u \in C([0, R]) ; a(R-t) \leq u(t) \leq b \text { for } 0 \leq t \leq R\} .
$$


Clearly, $W$ is a bounded closed convex subset of the Banach space $C([0, R])$ endowed with the sup norm. Define

$$
S u(t)=\int_{0}^{R} G_{n}(t, s)\left(\int_{0}^{R} G_{n}(s, r) f(u(r)) d r\right) d s
$$

for $t \in[0, R]$ and $u \in W$. Using $\left(\mathrm{H}_{1}\right)$ and the well-known properties of $G_{n}$ it is easily verified that $S$ is a compact operator mapping $W$ into itself, and so there exists $u \in W$ such that $u=S u$ by the Schauder fixed-point theorem. Clearly $u \in C^{4}([0, R])$ and $u$ is a solution of $\left(4.2^{\prime}\right)$.

\section{REFERENCES}

1. R. Dalmasso, Problème de Dirichlet homogène pour une équation biharmonique semi-linéaire dans une boule, Bull. Sci. Math. (2) 114 (1990), 123-137.

2. __ Symmetry properties of solutions of some fourth order ordinary differential equations, Bull. Sci. Math. (2) 117 (1993), 441-462.

3. D. R. Dunninger and M. Miclavcic, On a semilinear biharmonic equation, Nonlinear Anal. 16 (1991), 383-387.

4. L. A. Peletier and R. C. A. M. van der Vorst, Existence and nonexistence of positive solutions of nonlinear elliptic systems and the biharmonic equation, Differential Integral Equations 5 (1992), 747-767.

5. W. C. Troy, Symmetry properties in systems of semilinear elliptic equations, J. Differential Equations 42 (1981), 400-413.

Laboratoire LMC-IMAG, Equipe EDP, Tour IRMA, B. P. 53, F-38041, Grenoble Cedex 9, FRANCE

E-mail address: Robert.Dalmasso@imag.fr 